\title{
Classical and quantum scattering by a Coulomb potential
}

\author{
A.I.Nikishov * \\ I.E.Tamm Department of Theoretical Physics, \\ P.N.Lebedev Physical Institute, Moscow, Russia
}

October 29, 2018

\begin{abstract}
For relativistic energies the small angle classical cross section for scattering on a Coulomb potential agrees with the first Born approximation for quantum cross section for scalar particle only in the leading term. The disagreement in other terms can be avoided if the sum of all corrections to the first Born approximation for large enough Coulomb charge contain the classical terms which are independent of that charge. A small part of the difference in classical and quantum cross sections may be attributed to the fact that the relativistic quantum particle can rush through the field without interaction. We expect that smaller impact parameters and spin facilitate this affect.
\end{abstract}

\section{Introduction}

The initial motivation for this consideration was the interest in the properties of gravitational field. The heuristic approach to gravity [1-3] suggests that the Riemannian space appears as a result of changing by the gravitational field of measuring rods and clocks. If so, the formation of changes in the rods and clocks requires some time and something new can occur when the formation time become comparable with the period of gravitational frequency. In particular, one can imagine the situation when a relativistic particle can rush through the field so quickly that (with a non-zero probability) no interaction with the field occur.

Similar event can happen in a Coulomb scattering. In the nonrelativistic case the quantum particle scattering is governed by the same Rutherford formula as the classical one. In classical picture each particle is scattered by the field, so the above mentioned agreement should mean that passing through the field without interaction is impossible. In the relativistic region classical and quantum cross sections are different and a part of the difference may be attributed to the fact that quantum particle can fly through the field without being deflected. The classical cross section in small angle region have terms which have different signs for attractive and repulsive potentials. Their analogue in quantum case contain the Planck constant $\hbar$ in the denominator of the fine structure constant $\alpha=\frac{e^{2}}{\hbar c}$. This suggest that unless $\alpha Z$ is made sufficiently large the reproduction of classical terms from quantum ones is impossible. For this reason the theoretical investigation of the relationship between

*E-mail: nikishov@lpi.ru 
the classical and quantum scattering is of interest. It is even probable that some cleverly designed experiments can also clarify the situation.

\section{Scattering by attractive potential}

Now we are going to get the classical cross section. The trajectory of a classical particle in Coulomb field was obtained by C. G. Darwin in 1913 [4]. The scattering angle $\theta$ as a function of impact parameter $\rho$ and velocity at infinity $\beta=v / c$ is given by the relation

$$
\frac{\theta}{2}=\frac{\pi-\chi}{\sqrt{1-z^{-1}}}-\frac{\pi}{2}
$$

where

$$
\chi=\arctan \xi, \quad \xi=\beta \sqrt{z-1} \quad z=\left(\frac{\rho}{a}\right)^{2} \frac{\beta^{2}}{1-\beta^{2}}, \quad a=\frac{e e^{\prime}}{m c^{2}} .
$$

The integral cross section is

$$
\sigma_{c l}(\theta)=\pi \rho^{2}(\theta),
$$

because all particles with impact parameters less then $\rho$ are scattered, see Problem 1 in $\S 39$ in [5]. We note here that $\left(e e^{\prime}\right)^{2}$ enters into the classical cross section only as a factor $a^{2}=\left(\frac{e e^{\prime}}{m c^{2}}\right)^{2}$. This follows from (1) and (2) as $\rho / a$ is a function of only $\theta$ and $\beta$.

We expect that classical approach is justified for large angular momentum $\rho p$ ( $p$ is the

momentum at infinity) i.e. for large impact parameters. So we may assume that $\frac{\hbar}{\rho p}<<1$ is the accuracy of classical treatment. We can write

$$
\sqrt{z}=\frac{\rho p}{\hbar} \frac{\hbar c}{e e^{\prime}}
$$

We note that in the nonrelativistic region when $c \rightarrow \infty$ also $z \rightarrow \infty$. As seen from (4) the condition $\frac{\rho p}{\hbar}>>1$ can be satisfied even for $z$ of the order unity if $\frac{e e^{\prime}}{\hbar c}$ is correspondingly large.

In general we may write

$$
\frac{d \sigma_{c l}}{d \theta}=\pi \frac{1}{\left|\frac{d \theta}{d \rho^{2}}\right|},
$$

where $\frac{d \theta}{d \rho^{2}}$ should be calculated from (1) and (2) and taken at $\rho^{2}$ obtained numerically from (1) for the considered $\theta$.

For $\xi, z>>1$ we have

$$
\begin{gathered}
\chi \equiv \arctan \xi=\frac{\pi}{2}-\frac{1}{\xi}+\frac{1}{3 \xi^{3}}-\frac{1}{5 \xi^{5}}+\cdots, \\
\frac{1}{\xi}=\frac{1}{\beta \sqrt{z}}\left(1-z^{-1}\right)^{-1 / 2}=\frac{1}{\beta} \sqrt{\varepsilon}\left\{1+\frac{1}{2} \varepsilon+\frac{3}{2^{3}} \varepsilon^{2}+\cdots\right\}, \quad \varepsilon=\frac{1}{z} .
\end{gathered}
$$

From (6) it follows

$$
\chi=\frac{\pi}{2}-\beta^{-1} \sqrt{\varepsilon}+\left[-\frac{1}{2 \beta}+\frac{1}{3 \beta^{3}}\right] \varepsilon^{3 / 2}+\left[-\frac{3}{2^{3} \beta}+\frac{1}{2 \beta^{3}}-\frac{1}{5 \beta^{5}}\right] \varepsilon^{5 / 2}+\cdots .
$$

Using also

$$
\left(1-z^{-1}\right)^{-1 / 2}=\left\{1+\frac{1}{2} \varepsilon+\frac{3}{2^{3}} \varepsilon^{2}+\cdots\right\}
$$


we find for $\frac{\theta}{2}$ in $(1)$

$$
\frac{\theta}{2}=\frac{1}{\beta} \sqrt{\varepsilon}+\frac{1}{2^{2}} \pi \varepsilon+\left[\frac{1}{\beta}-\frac{1}{3 \beta^{3}}\right] \varepsilon^{3 / 2}+\frac{3}{2^{4}} \pi \varepsilon^{2}+\left[\frac{1}{\beta}-\frac{2}{3 \beta^{3}}+\frac{1}{5 \beta^{5}}\right] \varepsilon^{5 / 2}+\cdots
$$

This gives the scattering angle $\theta$ as a function of impact parameter $\rho$, see the expression for $z$ in $(2), \varepsilon=z^{-1}$.

Inverting (9) with the help of eq. (3.6.25) in [6] we obtain

$$
z^{-1 / 2}=\varepsilon^{1 / 2}=A \frac{\theta}{2}+B\left(\frac{\theta}{2}\right)^{2}+C\left(\frac{\theta}{2}\right)^{3}+D\left(\frac{\theta}{2}\right)^{4}+E\left(\frac{\theta}{2}\right)^{5}+\cdots
$$

where

$$
\begin{gathered}
A=\beta ; \quad B=-\frac{\pi}{2^{2}} \beta^{3} ; \quad C=\frac{1}{3} \beta-\beta^{3}+\frac{\pi^{2}}{2^{3}} \beta^{5} ; \quad D=-\frac{5 \pi}{2^{2} \cdot 3} \beta^{3}+\frac{17 \pi}{2^{4}} \beta^{5}-\frac{5 \pi^{3}}{2^{6}} \beta^{7} \\
E=\frac{2}{3 \cdot 5} \beta-\frac{2^{2}}{3} \beta^{3}+\left(2+\frac{7 \pi^{2}}{2^{4}}\right) \beta^{5}-\frac{3 \cdot 11}{2^{5}} \pi^{2} \beta^{7}+\frac{7 \pi^{4}}{2^{7}} \beta^{9} .
\end{gathered}
$$

Next we rewrite (10) in the form

$$
z^{-1 / 2}=\beta \frac{\theta}{2}\left\{1+a_{1} \frac{\theta}{2}++a_{2}\left(\frac{\theta}{2}\right)^{2}+a_{3}\left(\frac{\theta}{2}\right)^{3}+a_{4}\left(\frac{\theta}{2}\right)^{4}+\cdots\right\}
$$

From (12) with the help of eq. (3.6.17) in [6] we get

$$
\begin{gathered}
z=\left(\frac{2}{\beta \theta}\right)^{2} f(\theta / 2, \beta), \quad f(\theta / 2, \beta)=1+\frac{\pi \beta^{2}}{2} \frac{\theta}{2}+\left[-\frac{2}{3}+2 \beta^{2}-\frac{\pi^{2} \beta^{4}}{2^{4}}\right]\left(\frac{\theta}{2}\right)^{2}+ \\
+\left[\frac{\pi \beta^{2}}{3}-\frac{5 \pi \beta^{4}}{2^{3}}-\frac{\pi^{3} \beta^{6}}{2^{5}}\right]\left(\frac{\theta}{2}\right)^{3}+\left[\frac{1}{3 \cdot 5}+\frac{2 \beta^{2}}{3}-\left(1+\frac{\pi^{2}}{2^{2}}\right) \beta^{4}+\frac{3 \cdot 5 \pi^{2} \beta^{6}}{2^{5}}-\frac{5 \pi^{4} \beta^{8}}{2^{8}}\right]\left(\frac{\theta}{2}\right)^{4}+\cdots
\end{gathered}
$$

It follows from (2) that

$$
\rho^{2}=a^{2} \frac{1-\beta^{2}}{\beta^{2}} z
$$

Using here (13) we find

$$
\sigma_{c l}(\theta)=\pi \rho^{2}(\theta)=\pi\left(\frac{e e^{\prime}}{v p}\right)^{2} \frac{1}{(\theta / 2)^{2}} f(\theta / 2, \beta) ; \quad p=\frac{m v}{\sqrt{1-\beta^{2}}}
$$

where $f(\theta / 2, \beta)$ is given in (13). We see that terms of odd powers of $\theta$ are present in classical cross section. They have negative sign in the repulsive case, see below. This has clear physical explanation: in attractive case the particle comes closer to the center and is scattered in a larger angle. These terms are absent in quantum formula in Born approximation and the reason is also clear: the particle moves freely before and after a single interaction and the sign of particle's charge is unimportant in the cross section. So, using wave packets to fix the impact parameter will not change the situation. The correction to Born approximation do depend on the sign of particle's charge and is proportional to $\frac{e e^{\prime}}{\hbar c}$ i.e. to $\alpha Z$ and Planck constant is in the denominator. This suggests that the sum of all corrections can contain the 
classical terms if $\alpha Z$ is sufficiently large. The differential cross section is obtained from the integral one (15) by differentiation over $\theta$ (and changing the overall sign):

$$
\begin{aligned}
& \frac{d \sigma_{c l}}{d \theta}=\pi\left(\frac{e e^{\prime}}{v p}\right)^{2}\left\{\frac{8}{\theta^{3}}+\frac{\pi \beta^{2}}{\theta^{2}}+\left[-\frac{\pi}{6} \beta^{2}+\frac{5}{16} \pi \beta^{4}+\frac{1}{64} \pi^{3} \beta^{6}\right]+\right. \\
& \left.\left[-\frac{1}{30}-\frac{1}{3} \beta^{2}+\left(\frac{1}{2}+\frac{1}{8} \pi^{2}\right) \beta^{4}-\frac{15}{64} \pi^{2} \beta^{6}+\frac{5}{512} \pi^{4} \beta^{8}\right] \theta+\cdots\right\} .
\end{aligned}
$$

Now we can compare the classical cross section with the quantum one for a scalar particle

$$
\begin{gathered}
\sigma_{q u}(\theta)=\pi\left(\frac{e e^{\prime}}{v p}\right)^{2} \int_{\theta}^{\pi} \frac{\cos (\theta / 2)}{\sin ^{3}(\theta / 2)} d \theta=\pi\left(\frac{e e^{\prime}}{v p}\right)^{2} \cot ^{2}(\theta / 2)= \\
\pi\left(\frac{e e^{\prime}}{v p}\right)^{2} \frac{1}{(\theta / 2)^{2}}\left\{1-\frac{2}{3}\left(\frac{\theta}{2}\right)^{2}+\frac{1}{3 \cdot 5}\left(\frac{\theta}{2}\right)^{4}+\cdots\right\},
\end{gathered}
$$

see, for example [7]. We note that $f(\theta / 2, \beta)$ for $\beta \rightarrow 0$ goes over to the expression in braces in the right hand side of (17). This have to be expected as (17) is essentially the (integral) Rutherford cross section.

Finally, the coefficient in front of $(\theta / 2)^{4}$ is larger in classical cross section than in quantum one. It is enticing to interpret this in such a way the that the quantum particle can fly through

the field without interaction. Such a possibility is of great interest for gravitational field where the analogue of $e e^{\prime}$ is $G m m^{\prime}$ and is not restricted.

If the scattered particle have spin $1 / 2$ the quantum cross section acquires the additional factor $\left(1-\beta^{2} \sin (\theta / 2)\right)$, see eq. (7.22) in [8]. It looks like the spin of a particle and smaller impact parameters (corresponding to larger $\theta /$ ) facilitate the flight without interaction.

Returning to equation (17), we note that the integrand gives the differential cross section and

$$
\frac{\cos (\theta / 2)}{\sin ^{3}(\theta / 2)}=\frac{8}{\theta^{3}}-\frac{1}{30} \theta+\cdots
$$

For the electron we have

$$
\frac{\cos (\theta / 2)}{\sin ^{3}(\theta / 2)}\left(1-\beta^{2} \sin ^{2}(\theta / 2)\right)=\frac{8}{\theta^{3}}-\frac{2 \beta^{2}}{\theta}+\left(\frac{\beta^{2}}{6}-\frac{1}{30}\right) \theta+\cdots .
$$

\section{Scattering by repulsive potential}

In this case instead of (1) we have

$$
\frac{\theta}{2}=\frac{\pi}{2}-\frac{\chi}{\sqrt{1-z^{-1}}}
$$

see Problem 1 in $§ 39$ in [5]. (The angle $\varphi_{0}$ in [5] is the half angle between asymptotes, not the whole angle as misprinted there.) In the same manner as before we obtain (10), where $A, C$ and $E$ are the same as in (11), but $B$ and $D$ have opposite sign, i.e. they are obtained from (11) by substitution: $B \rightarrow-B, D \rightarrow-D$. Correspondingly in the expression for $z$ in (13) and in (15) we have to make the substitution $\theta \rightarrow-\theta$ and in (16) also $d \theta \rightarrow-d \theta$. 
We note also that (3) simply states that each classical particle with the impact parameter $\rho<\rho(\theta)$ is scattered. The differential cross section is obtained from the integral one in (3) by differentiating over $\theta$ (and changing the sign). For $\theta$ not small enough the classical approach is inapplicable, but the integral cross section should be valid as the "total" integral cross section (for small $\theta$ ) in the sense that it include inelastic processes (as bremsstrahlung) and absorption by some disk around Coulomb center.

\section{Conclusions}

The classical cross section for scattering in relativistic region is different for attractive and repulsive potentials and do not agrees exactly with quantum cross section for scalar particle. It seems that this disagreement cannot be totally ascribed to the fault of classical approach. This suggest that quantum corrections to the first Born approximation should have such a structure that for sufficiently large $\alpha Z$ they give classical terms which are independent of $\alpha Z$.

It is not excluded that with small probability a high energy particle can rush through Coulomb field without deflection.

\section{Acknowledgements}

I am greatly indebted to V.I. Ritus for fruitful discussions.

The work was carried out with financial support of Scientific Schools and Russian Fund for Fundamental Research (Grants 4401.2006.2 and 05-02-17217).

\section{References}

1. Dehnen H., Hönl H., and Westpfahl K., Ann. der Phys. 6, 7 Folge, Band 6, Heft 7-8, S. 370-407, (1960).

2. Thirring W.E., Ann. Phys. (N.Y.) 16, 96-117,(1961).

3. Nikishov A.I. arXiv:0710.4445, v1 [gr-qc] 24 Oct 2007.

4. Darwin C.G. Phil. Mag.(6) 25, p. 201 (1913).

5.Landau L.D. add Lifshitz E.M. The Classical theory of Fields, Cambridge, MA (1971)

6.Abramowitz M. and Stegun I. Handbook of Mathematical Functions, National Bureau of Standards (1964).

7. Pauli W. Rev. Mod. Phys. 13, 203-232 (1941).

8. Bjorken J.D. and Drell S.D. Relativistic Quantum Mechanics. McGraw-Hill Book Company, New York (1964). 